\title{
Hypobaric-Ischemic Conditions Produce Glutamate-like Cytopathology in Infant Rat Brain
}

\author{
C. Ikonomidou, M. T. Price, J. L. Mosinger, G. Frierdich, J. Labruyere, K. Shahid Salles, and J. W. Olney \\ Department of Psychiatry, Washington University School of Medicine, St. Louis, Missouri 63110
}

\begin{abstract}
We present a new animal model of perinatal hypoxic/ischemic brain damage and compare this type of brain damage with the excitotoxic type of damage previously described in the brains of infant rats and monkeys treated systemically with glutamate (Glu). Ten-d-old rats with unilateral occlusion of the common carotid artery were subjected to hypobaric conditions for $\mathbf{7 5} \mathrm{min}$ and sacrificed $\mathbf{0 - 4} \mathrm{hr}$ later for light and electron microscopic brain examination. The mortality rate was relatively low $(12 \%)$, and brain damage was evident ipsilateral to the ligated carotid in $94 \%$ of surviving animals $4 \mathrm{hr}$ after termination of the hypobaric event. Regions most frequently affected were the medial habenulum, dentate gyrus, caudate nucleus, frontoparietal neocortices, olfactory tubercle, and several thalamic nuclei. The acute cytopathological changes, primarily edematous degeneration of neuronal dendrites and cell bodies, evolved very rapidly, with some neurons manifesting end-stage necrosis at $0 \mathrm{hr}$ (immediately after hypobaric exposure) and others developing such changes over a 1-4-hr period. We conclude that the neurodegenerative reaction induced in infant rat brain by hypoxia/ischemia is indistinguishable from the excitotoxic type of damage exogenous Glu is known to cause. Moreover, in a companion study (Olney et al., 1989) we show that MK801 , a powerful antagonist of the $\mathrm{N}$-methyl-D-aspartate receptor complex (subtype of Glu receptor), protects against neuronal degeneration in this hypobaric/ischemic model. Our results reinforce other recent evidence suggesting that hypoxic/ischemic brain damage is mediated by endogenous Glu or related excitotoxins.
\end{abstract}

Glutamate (Glu) and aspartate (Asp) are major excitatory transmitters (Watkins, 1978) and neurotoxins (excitotoxins) that have the potential to destroy central neurons by an excitatory mechanism (Olney, 1969a; Olney et al., 1971). Subcutaneous or oral administration of Glu to infant rodents or monkeys induces acute neuronal necrosis in certain brain regions that lack blood brain barriers (Olney 1969a, 1971; Olney and Ho, 1970; Olney et al., 1972). The neurodegenerative process is exceedingly acute; vulnerable neurons display swelling within 15-30 min after Glu administration and progress to end-stage necrosis over a 1-4hr period (Olney 1971; Olney et al., 1971, 1972). Recent evidence suggests that endogenous Glu and/or Asp may be re-

Received Aug. 19, 1988; revised Oct. 3, 1988; accepted Oct. 7, 1988.

Supported in part by NIMH Research Scientist Award MH 38894 (J.W.O.), HHS grants HD 24237 and ES 07066, and a grant from Pfizer Pharmaceutical.

Correspondence should be addressed to John W. Olney, M.D., Department of Psychiatry, Washington University School of Medicine, 4940 Audubon Ave., St. Louis, MO 63110.

Copyright (C) 1989 Society for Neuroscience $0270-6474 / 89 / 051693-08 \$ 02.00 / 0$ sponsible for hypoxic/ischemic neuronal degeneration in conditions such as stroke, cardiac arrest, and perinatal asphyxia (Benveniste et al., 1984; Rothman, 1984; Simon et al., 1984; Weiss et al., 1986; Gill et al., 1987; Lawrence et al., 1987; McDonald et al., 1987; Olney et al., 1987, 1988; Prince and Freeser, 1988). If endogenous Glu is the responsible pathogenic agent in hypoxic/ischemic brain damage, it should be possible to show that hypoxia/ischemia causes the same type of cytopathological changes in brain that exogenous Glu typically causes. Here we describe a new model of hypoxic/ischemic brain damage in the infant rat and provide light and electron microscopic evidence that the acutely evolving cytopathology associated with hypoxia/ischemia is indistinguishable from that induced by exogenous Glu.

\section{Materials and Methods}

Our method for producing hypoxic/ischemic brain damage in infant rats is similar to that described by Levine (1960) as modified by Rice et al. (1980), but it involves exposure of infant animals to a partial vacuum rather than an environment of reduced oxygen and increased nitrogen. Ten-d-old Sprague-Dawley rats were used in all experiments. A unilateral carotid ligation was performed under halothane anesthesia. During and immediately after surgery the pups were kept on a warming pad that maintained their body temperature at approximately $36^{\circ} \mathrm{C}$ as measured by a skin surface microprobe. After recovery from anesthesia, a group of 10-12 pups (total body wt $=200 \perp 10 \mathrm{gm}$ ) was placed in a hypobaric chamber that measured $2370 \mathrm{ml}$ in volume and was immersed in a water bath that maintained the temperature inside the chamber at $36 \pm 0.5^{\circ} \mathrm{C}$. The air pressure in the chamber was gradually reduced from 760 to $225 \mathrm{~mm} \mathrm{Hg}$ over a 1-min period and maintained at $225 \mathrm{~mm}$ for $75 \mathrm{~min}$, then brought back to $760 \mathrm{~mm}$ over a 1 -min period. Either immediately or 1-4 hr after removal from the hypobaric chamber, the rats were anesthetized with chloral hydrate and perfused through the heart and ascending aorta with a solution of paraformaldehyde $(1 \%)$ and glutaraldehyde $(1.5 \%)$ in phosphate buffer. In some cases the neck incision was opened prior to perfusion and the suture removed from the carotid artery (a piece of polyethylene tubing originally placed between the suture and vessel wall facilitated removal of the suture without damage to the vessel); however, this was not found ncccssary in order to achieve adequate preservation of the brain. After perfusion for $15 \mathrm{~min}$ the brains were removed and sliced in $1-\mathrm{mm}$ transverse slabs that were additionally fixed in osmium tetroxide (1\%), dehydrated in graded ethanols, cleared in toluene, and embedded in araldite. Sections $1 \mu \mathrm{m}$ thick were cut with $1 / 2^{\prime \prime}$ glass knives at $40-\mu \mathrm{m}$ intervals on a Sorval MT-2B ultratome, stained with methylene blue/ azure II, and evaluated by light microscopy. Ultrathin sections were cut from areas of special interest from the same blocks, stained with lead citrate and uranyl acetate, and examined with a JEOL 100 B transmission electron microscope. These histological methods are the same as were used in previous studies of Glu-induced brain damage (Olney, 1971; Olney et al., 1972).

\section{Results}

In pilot experiments, it was observed that unilateral carotid ligation alone did not induce brain damage, nor did exposure 

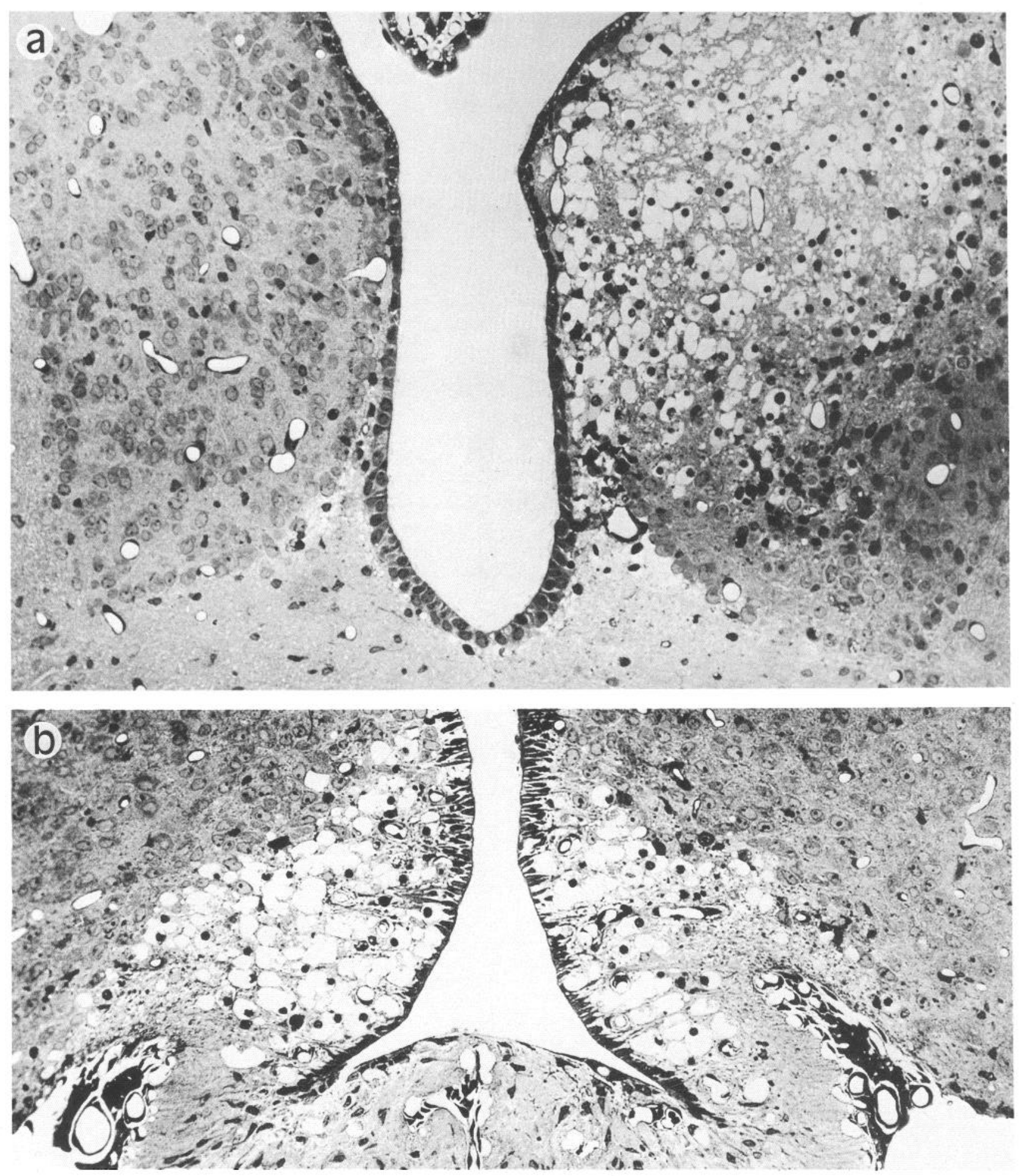

Figure 1. a, Medial habenular nucleus of a 10-d-old rat subjected to unilateral carotid ligation and 75 min in a hypobaric chamber followed by 4-hr recovery. The medial habenulum, a bilateral nucleus, exhibits damage unilaterally on the side ipsilateral to the carotid ligation. $b$, Arcuate hypothalamic nucleus of a 21 -d-old mouse that had ingested a solution containing $10 \%$ glutamate 4 hr previously. Arcuate neurons are destroyed in a bilaterally symmetrical pattern. Note that the hypobaric/ischemic cytopathology $(a)$ appears identical to that induced by exogenous glutamate (b). Degenerating neurons in each case typically have swollen edematous cytoplasm and dark pyknotic nuclei. Magnification, $\times 200$.

to the above hypobaric conditions. Among all animals in the present study that were allowed to survive $2-4 \mathrm{hr}(n=109)$, the mortality rate was $12 \%$ (death having occurred in the hypobaric chamber), and the overall incidence of brain damage among survivors was $94 \%$. In all cases, the damage was unilaterally restricted to the side of the carotid ligation. In a group of 31 animals randomly selected for comprehensive histological evaluation, the distribution of lesions and frequency with which 
each brain region was involved is given in Table 1. A striking lesion was consistently found in the medial habenulum (Fig. 1). Also quite consistently, we found a cytopathological reaction affecting granule neurons in the ventral blade of the dentate gyrus immediately overlying the damaged medial habenulum. Other regions frequently damaged (Fig. 2) were the frontal and parietal neocortices, caudate nucleus, medial dorsal and lateral dorsal nuclei of the thalamus, olfactory tubercle, dorsal subiculum, and islands of Calleja.

The acute cytopathological changes appeared by light microscopy to consist predominantly of edematous swelling of dendritic processes (confirmed ultrastructurally; see below) and acute edematous degeneration of neuronal cell bodies with pyknotic changes occurring quite early in the nucleus. Comparison of the acute cytopathological reaction in the hypothalamus of a mouse treated orally with Glu and that in the medial habenulum of an infant rat subjected to hypobaric/ischemic conditions (Fig. 1) demonstrates that the 2 cytopathological processes are indistinguishable at the light microscopic level. Somc neurons, primarily in the hippocampus, cortex, thalamus, and caudate nucleus, underwent a more slowly developing process of vacuolar condensation in which both the cytoplasm and the nucleoplasm became uniformly dense and dark-staining except for numerous spherical vacuoles in the cytoplasm. This type of degenerative reaction, sometimes referred to as "coagulative" degeneration, has been described previously as a feature of the cytopathology associated with cerebral ischemia (Van Reempts, 1984), status epilepticus (Olney et al., 1986a), and systemic administration of Glu to infant rodents or monkeys (Olney, 1969a, 1986).

To study the time course of the acutely evolving lesion, we killed infant rats at intervals from 0 to $4 \mathrm{hr}$ post-hypoxia. Surprisingly, animals killed at $0 \mathrm{hr}$ (immediately after removal from the hypobaric chamber) consistently displayed cytopathological changes, including cnd-stage ncuronal nccrosis. In fact, onc fcature of the cytopathology, dendritic swelling, was more extensive in certain brain regions at $0 \mathrm{hr}$ than at $4 \mathrm{hr}$. The other major feature, degeneration of cell bodies (both edematous degeneration and vacuolar condensation), was more extensively developed at $4 \mathrm{hr}$.

By electron microscopy, it was readily confirmed that the major tissue components initially affected were neuronal dendrites (Fig. 3) and cell bodies (Fig. 4); indeed, every feature of the acute cytopathology in either dendritic or somal neural elements was identical to that previously described (Olney, 1971; Olney et al., 1972) in infant rodent or monkey brain following systemic administration of exogenous Glu (Figs. 3, 4). In nerve cells destined to undergo acute edematous degeneration, mitochondria exhibited the first sign of pathological change; before cytoplasmic swelling occurred, mitochondria became transiently condensed, with their membranes assuming a thickened, dark, compacted appearance (Fig. 5). Subsequently, as signs of edematous degeneration began to pervade the entire cytoplasmic compartment, mitochondria became swollen (Fig. 5). Finally, in later stages of neuronal necrosis, mitochondria appeared less swollen and typically displayed a small and spherical ("roundedup") profile (Fig. 4). These several stages of mitochondrial transformation could be detected simultaneously in a given brain. For example, the lesion affecting dentate granule neurons tended to develop more slowly than the lesion affecting medial habenular neurons. Thus, at 0 to $1 \mathrm{hr}$ it was possible to find swollen mitochondria in medial habenular neurons that were undergo-
Table 1. Frequency of damage in various brain regions

\begin{tabular}{ll} 
Brain region & Frequency (\%) \\
\hline Habenular nucleus, medial & 94 \\
Dentate gyrus (granule cells) & 81 \\
Caudate nucleus & 71 \\
Thalamus, mediodorsal nucleus & 71 \\
Neocortex, parietal & 65 \\
Olfactory tubercle & 63 \\
Subiculum, dorsal & 61 \\
Thalamus, lateral dorsal nucleus & 58 \\
Islands of Calleja & 56 \\
Neocortex, frontal & 52 \\
Septum & 38 \\
Hippocampus, CA1 pyramids & 35 \\
Thalamus, ventrobasal nucleus & 32 \\
Habenular nucleus, lateral & 32 \\
Hippocampus, CA3 pyramids & 16 \\
Pyriform cortex & 10 \\
Amygdala, cortical nucleus & 7 \\
\hline
\end{tabular}

ing edematous degeneration and condensed mitochondria in dentate granule neurons that had not yet begun manifesting edematous degeneration (Fig. 5). But at $4 \mathrm{hr}$, dentate granule neurons exhibited edematous degeneration and contained $\mathrm{mi}$ tochondria that displayed swollen profiles (Fig. 5). By this time ( $4 \mathrm{hr}$ ), medial habenular neurons were in an advanced necrotic stage, and their mitochondria had become small and spherical (Fig. 4). Other features of the edematous degenerative reaction affecting the cytoplasmic compartment of neuronal cell bodies included dissolution of the endoplasmic reticular system with formation of spherical vacuoles bounded by rough membranes, disaggregation of polysomes, and dispersal of nondescript particulate material in the cytoplasm (Fig. 4). Changes in the neuronal nucleus typically consisted of chromatin clumping with the clumps finally coalescing centrally to form a pyknotic nucleus (Fig. 4).

The evacuation of degenerating elements was very rapid in periventricular regions, especially the medial habenulum. Four hours after hypobaric exposure non-neuronal phagocytic cells were enveloping and incorporating neuronal cell bodies, and within $24 \mathrm{hr}$ much of the evacuation process had transpired. In the thalamus and neocortex, biological debridement proceeded more slowly and was still evident as an ongoing process $4 \mathrm{~d}$ post-ischemia.

\section{Discussion}

We have shown that unilateral ligation of the common carotid artery and subsequent exposure of 10-d-old rat pups to a partial vacuum causes a reproducible pattern of neuronal degeneration in several brain regions ipsilateral to the ligation. We also have studied brains of infant rats subjected to the hypoxic/ischemic protocol described by Rice et al. (1980), which consists of unilateral common carotid artery ligation followed by $2-3 \mathrm{hr}$ in a reduced oxygen environment, and demonstrated (Olney, 1988) that the cytopathological changes induced by that approach are identical to the hypobaric/ischemic changes described here. However, with this approach we were unable to find a set of conditions yielding a consistently high incidence of lesions without an unacceptably high mortality rate. We abandoned this 



Figure 2. Ten-d-old infant rat brain $4 \mathrm{hr}$ after the animal was subjected to hypobaric/ischemic conditions. Neurons in the parietal cortex contralateral to the carotid ligation have a normal appearance $(a)$. Ipsilateral to the carotid ligation, acutely degenerating neurons in the parietal cortex $(b)$, caudate nucleus $(c)$, and olfactory tubercle $(d)$ display changes that are indistinguishable from those induced by exogenous Glu (Fig. $1 b$ ). Magnification, 



Figure 3. a, Swollen dendrite $(D)$ undergoing acute edematous degeneration in the medial dorsal nucleus of the thalamus of a 10 -d-old rat 4 hr after hypobaric/ischemic exposure. This degenerating dendrite is in contact with a presynaptic axon terminal $(A)$, which retains a normal appearance. This cytopathology is typical of Glu-induced damage as shown in $b$, where a swollen dendrite $(D)$ is undergoing acute edematous degeneration in the arcuate hypothalamic nucleus of an infant rhesus monkey $4 \mathrm{hr}$ after oral administration of Glu $(0.5 \mathrm{~g} / \mathrm{kg})$; note the presence of a presynaptic axon terminal $(A)$ that retains a normal appearance. Magnification, $\times 27,000$.

model after finding that the hypobaric/ischemic approach yielded more favorable results regarding lesion incidence and mortality rate while simultaneously being easier and less expensive and entailing less stress (shorter period of hypoxia) for the experimental animal.

Brain damage that occurs during the perinatal period in the human is often referred to as hypoxic/ischemic because typically it appears to be caused by a combination of reduced oxygen content of blood and regional reductions in cerebral blood flow (Johnston and Silverstein, 1987). A pathological sequence is thought to occur in which anoxia triggers systemic hypotension and reduced cardiac output, which may be further complicated by failure of cerebral blood vessels to autoregulate under anoxic conditions (Lou et al., 1979). The brain damage model described here can well be characterized as hypoxic/ischemic, since low blood oxygen tension coupled with inadequate vascular perfusion of the affected side of the brain is the primary factor contributing to its occurrence. Perinatal brain damage in the human and hypobaric/ischemic brain damage in the infant rat have additional features in common. In human infants dying during or shortly after a hypoxic episode, histological changes characterized by swollen vacuous cytoplasm and clumping or karyorrhexis of nuclear chromatin have been described (Banker and Larroche, 1962). Neurons that are sensitive to hypoxic/ischemic damage in the infant human include those in the cerebral cortex, basal ganglia, various thalamic nuclei, and certain periventricular zones (Banker and Larroche, 1962; Brierley and Graham, 1984). These regions are also consistently affected in the hypobaric/ischemic infant rat brain. It has been proposed that vulnerability of periventricular regions in the immature human brain relates to a lag in development of ventriculopetal arteries that are destined to supply these regions (Banker and Larroche, 1962). Conceivably, this might account for the extreme vulnerability of medial habenular neurons to hypobaric/ischemic injury observed in the present study. Selective degeneration of other neuronal groups might be explained in the rat model by the watershed phenomenon, a concept often invoked to explain human neuropathology, in which neurons that lie between distal tributaries of separate arterial trunks are hypervulnerable to degeneration under hypoxic/ischemic circumstances. Further cross-species comparisons may not be warranted, since the pattern of neuropathology resulting from hypoxic/ischemic conditions may vary as a function of developmental age at the time of insult and it is difficult to know exactly what point in human ontogenesis corresponds with the tenth postnatal day in the rat.

Several lines of recent evidence support the hypothesis that hypoxic/ischemic brain damage is mediated by endogenous Glu or a related excitotoxin. Ischemia of the adult rat brain is as- 

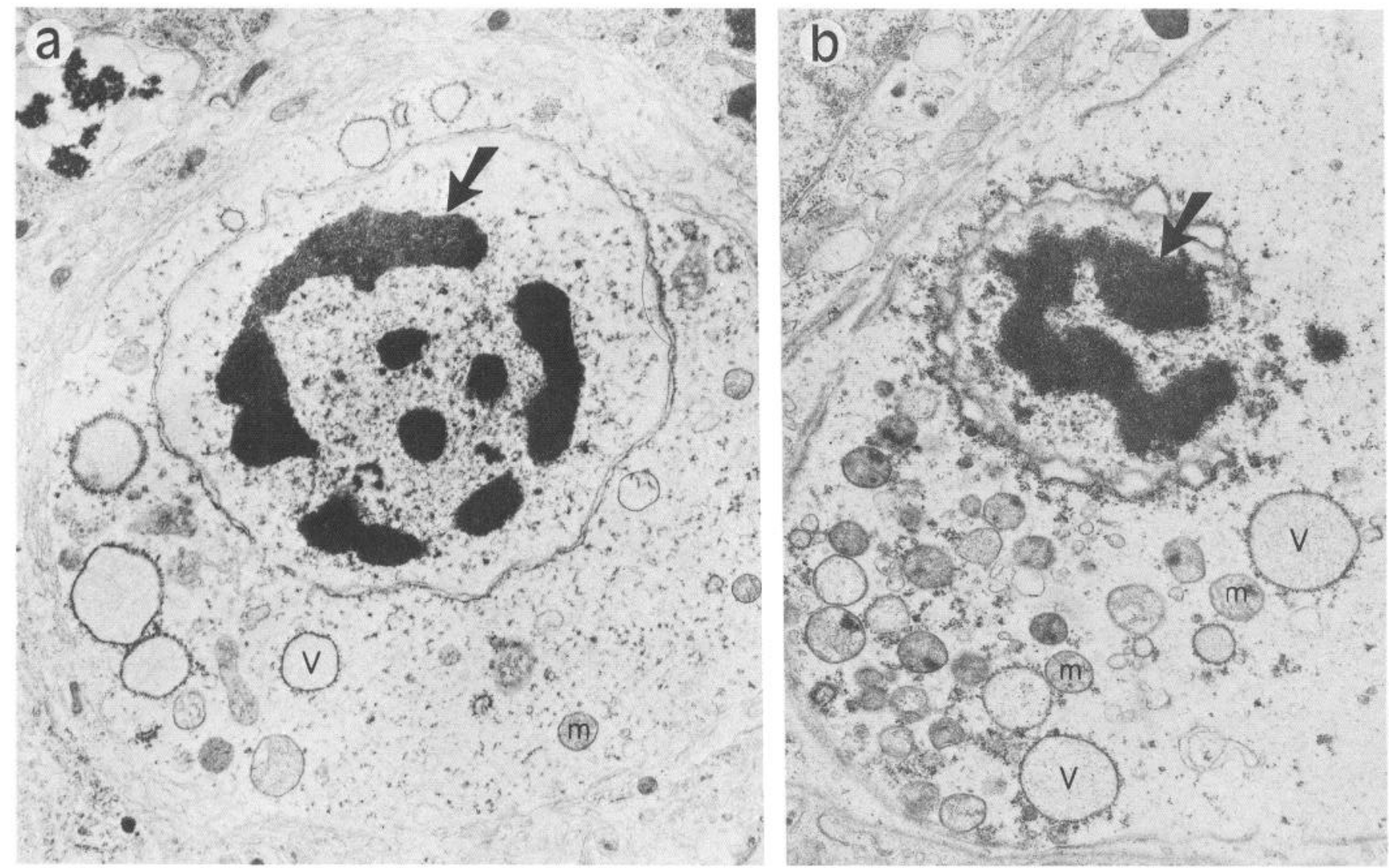

Figure 4. a, Neuron undergoing acute degeneration in the medial habenular nucleus of the 10-d-old rat $4 \mathrm{hr}$ after hypobaric/ischemic exposure. $b$, neuron undergoing acute degeneration in the arcuate hypothalamic nucleus of an infant rhesus monkey $4 \mathrm{hr}$ after oral administration of Glu (1 $\mathrm{g} / \mathrm{kg}$ ). Note the typical features of Glu-induced neuronal degeneration in both figures, including vacuolated endoplasmic reticulum ( $v$ ), spherical mitochondria $(\mathrm{m})$, and clumped chromatin (arrow) that is consolidating centrally to produce the typical appearance of a pyknotic nucleus. The hypobaric/ischemic changes exhibited by the neuron in $a$ are indistinguishable from those induced by Glu (b). Magnification, $\times 20,000$.

sociated with an accumulation of Glu and Asp in the extracellular compartment, where these agents can exert excitotoxic activity at postsynaptic dendrosomal receptors (Benveniste et al., 1984). The uptake mechanism for removal of Glu and Asp from the extracellular compartment is impaired during hypoxia/ ischemia in the infant rat brain (Silverstein et al., 1986). Transection of the excitatory (probably glutamergic) afferents to the hippocampus protects hippocampal neurons from hypoxic/ischemic damage (Wieloch et al., 1985). EAA antagonists reportedly protect against anoxic and/or ischemic neuronal degeneration in cell cultures of hippocampal neurons (Rothman, 1984) or cerebrocortical neurons (Weiss et al., 1986), in the ex vivo chick embryo retina (Olney et al., 1987), in the in vivo adult hippocampus of the rat (Simon et al., 1984) and gerbil (Gill et al., 1987; Lawrence et al., 1987), and in several regions of the in vivo infant rat brain (McDonald et al., 1987; Prince and Freeser, 1988).

A side-by-side comparison of the hypobaric/ischemic neurodegenerative reaction in infant rat brain with that induced in immature rodent or monkey brain by systemic administration of exogenous Glu reveals many similarities. In both syndromes postsynaptic dendrosomal neural elements are preferentially affected, with conspicuous sparing of presynaptic axons and identical changes occurring in cytoplasmic organelle systems and nuclear chromatin. Moreover, the time course of the neurodegenerative reaction is often remarkably acute, with the most sensitive neurons undergoing degenerative changes within minutes and progressing to end-stage necrosis within a few hours after either Glu administration or exposure to hypobaric/ischemic conditions. A less acute in vivo neurodegenerative process following Glu administration has also been described (Olney, $1969 b, 1986)$ in which the neuronal cytoplasm becomes vacuolated and the entire cell assumes a condensed "dark cell" appearance. In the present study, a similar rather protracted "dark cell" degenerative reaction was seen in several regions of the infant rat brain following exposure to hypobaric/ischemic conditions. Recently, 2 types of excitotoxic reaction to Glu have been described in in vitro preparations: an exceedingly acute process (Rothman, 1985; Olney et al., 1986b) that is not dependent on $\mathrm{Ca}^{2+}$ influx, and a delayed process (Choi, 1987) that is $\mathrm{Ca}^{2+}$ dependent. Although the present study does not address the issue of $\mathrm{Ca}^{2+}$ dependency, it documents that hypoxic/ischemic neuronal degeneration, like Glu-induced neuronal degeneration, may have either an acute or a delayed time course. Thus, both on the basis of pathomorphological criteria and time course, the neurodegenerative reaction to hypobaric/ischemic conditions in the infant rat brain is indistinguishable from the well-known reaction of CNS neurons to exogenous Glu.

One of our motivations for developing the infant rat preparation described here was to have an hypoxic/ischemic model in which neurons clearly manifest the remarkably acute type of dendrosomal pathology that traditionally has been associated 



Figure 5. Dentate granule neurons in the 10-d-old infant rat brain at $0 \mathrm{hr}(a)$ and $4 \mathrm{hr}(b)$ after hypobaric/ischemic exposure. $a$, Very early stage of degeneration, in which the only detectable sign of pathological change is the dense, compacted appearance of the mitochondria (arrow). $b$, Later stage of degeneration, in which the cytoplasmic compartment is becoming edematous, nuclear chromatin is beginning to form coarse clumps, and mitochondria (arrows) are spherical, edematous, and swollen. Each of these types of mitochondrial change has been described as a typical feature of Glu-induced neuronal degeneration (Olney, 1986). Magnification, ×45,000.

with Glu toxicity. In addition, since much of the in vivo research pertaining to the role of excitotoxins in hypoxic/ischemic neuronal degeneration (Simon et al., 1984; Gill et al., 1987; Lawrence et al., 1987) has focused exclusively on a single type of neuron, the CA1 hippocampal pyramidal neuron, there is a need for new models pertaining to other neuronal populations. Finally, in order to determine whether anti-excitotoxic drugs can prevent hypoxic/ischemic neuronal degeneration, it is essential to have a model in which the same degree and pattern of brain damage are consistently reproduced and in which the mortality rate is low. The model described here meets the several criteria sought; that this model may be of value for studying methods of preventing perinatal hypoxic/ischemic brain damage is corroborated in a companion article (Olney et al., 1989).

\section{References}

Banker, B. Q., and J. C. Larroche (1962) Periventricular leukomalacia in infancy: A form of neonatal anoxic encephalopathy. Arch. Neurol. 7: $386-410$

Benveniste, H., J. Drejer, A. Schousboe, and N. H. Diemer (1984) Elevation of extracellular concentrations of glutamate and aspartate in rat hippocampus during transient cerebral ischemia monitored by intracerebral microdialysis. J. Neurochem. 43: 1369-1374.
Brierley, J. B., and D. I. Graham (1984) Hypoxia and vascular disorders of the central nervous system. In Greenfield's Neuropathology, J. H. Adams, J. A. N. Corsellis, and L. W. Duchen, eds., pp. 125207, Arnold, London.

Choi, D. W. (1987) Ionic dependence of glutamate neurotoxicity. J. Neurosci. 7: 369-379.

Gill, R., A. C. Foster, and G. N. Woodruff (1987) Systemic administration of MK-801 protects against ischemia-induced hippocampal neurodegeneration in the gerbil. J. Neurosci. 7: 3343-3349.

Johnston, M. V., and F. S. Silverstein (1987) Perinatal anoxia. In Animal Models of Dementia, pp. 223-251, Liss, New York.

Lawrence, J J., T. A. Fuller, and J. W. Olney (1987) MK-801 and PCP protect against ischemic neuronal degeneration in the gerbil hippocampus. Soc. Neurosci. Abstr. 13: 1079.

Levine, S. (1960) Anoxic-ischemic encephalopathy in rats. Am. J. Pathol. 36: 1-17.

Lou, H. C., N. A. Lassen, and B. Friis-Hansen (1979) Impaired autoregulation of cerebral blood flow in the distressed newborn. J. Pediatr. 94: 118-127.

McDonald, J. W., F. S. Silverstein, and M. V. Johnston (1987) MK801 protects the neonatal brain from hypoxic/ischemic damage. Eur. J. Pharmacol. 140: 359-361.

Olney, J. W. (1969a) Brain lesions, obesity and other disturbances in mice treated with monosodium glutamate. Science 164: 719-721.

Olney, J. W. (1969b) Glutamate-induced retinal degeneration in neonatal mice. Electron microscopy of the acutely evolving lesion. J. Neuropathol. Exp. Neurol. 28: 455-474.

Olney, J. W. (1971) Glutamate-induced neuronal necrosis in the infant 
mouse hypothalamus: An electron microscopic study. J. Neuropathol. Exp. Neurol. 30: 75-90.

Olney, J. W. (1986) Inciting excitotoxic cytocide among central neurons. In Excitatory Amino Acids and Epilepsy, R. Schwarcz and Y. Ben-Ari, eds., pp. 631-645, Plenum, New York.

Olney, J. W. (1988) Endogenous excitotoxins and neuropathological disorders. In Excitatory Amino Acids in Health and Disease, D. Lodge, ed., pp. 337-351, Wiley, London.

Olney, J. W. and O. L. Ho (1970) Brain damage in infant mice following oral intake of glutamate, aspartate or cysteine. Nature 227: 609-610.

Olney, J. W., O. L. Ho, and V. Rhec (1971) Cytotoxic cffcets of acidic and sulphur-containing amino acids on the infant mouse central nervous system. Exp. Brain Res. 14: 61-76.

Olney, J. W., L. G. Sharpe, and R. D. Feigin (1972) Glutamate-induced brain damage in infant primates. J. Neuropathol. Exp. Neurol. 31: 464-488.

Olney, J. W., R. C. Collins, and R. S. Sloviter (1986a) Excitotoxic mechanisms of epileptic brain damage. Adv. Neurol. 44: 857-877.

Olney, J. W., M. T. Price, L. Samson, and J. Labruyere (1986b) The role of specific ions in glutamate neurotoxicity. Neurosci. Lett. 65 : $65-71$.

Olney, J. W., M. T. Price, J. Labruyere, E. Silverman, and M. Mueller (1987) Comparative efficacy of various agents in preventing glutamate-induced or ischemic neuronal degeneration in chick retina. Soc. Neurosci. Abstr. 13: 1030.

Olney, J. W., C. Ikonomidou, J. L. Mosinger, and G. Frierdich (1989) MK-801 prevents hypobaric-ischemic neuronal degeneration in infant rat brain. J. Neurosci. 9: 1701-1704.
Prince, D. A., and H. R. Freeser (1988) Dextromethorphan protects against cerebral infarction in a rat model of hypoxia-ischemia. Neurosci. Lett. 85: 291-296.

Rice, J. E., R. C. Vannuci, and J. B. Brierley (1980) The influence of immaturity on hypoxic-ischemic brain damage in the rat. Ann. Neurol. 9: 131-141.

Rothman, S. M. (1984) Synaptic release of excitatory amino acid neurotransmitter mediates anoxic neuronal death. J. Neurosci. 4: 18841891 .

Rothman, S. M. (1985) The neurotoxicity of excitatory amino acids is produced by passive chloride influx. J. Neurosci. 5: 1483-1489.

Silverstein, F. S., K. Buchanan, and M. V. Johnston (1986) Perinatal hypoxia-ischemia disrupts striatal high affinity $\left[{ }^{3} \mathrm{H}\right]$-glutamate uptake into synaptosomes. J. Neurochem. 47: 1614-1619.

Simon, R. P., J. H. Swan, T. Griffiths, and B. S. Meldrum (1984) Blockade of N-methyl-D-aspartate receptors may protect against ischemic damage in the brain. Science 226: 850-853.

Van Reempts, J. (1984) The hypoxic brain: Histological and ultrastructural aspects. Behav. Brain Res. 14: 99-108.

Watkins, J. C. (1978) Excitatory amino acids. In Kainic Acid as a Tool in Neurobiology, E. McGeer, J. W. Olney, and P. McGeer, eds., pp. 37-69, Raven, New York.

Weiss, J., M. P. Goldberg, and D. W. Choi (1986) Ketamine protects cultured neocortical neurons from hypoxic injury. Brain Res. 380: $186-190$

Wieloch, T., O. Lindrall, P. Blomqvist, and F. Gage (1985) Evidence for amelioration of ischemic neuronal damage in the hippocampal formation by lesions of the perforant path. Neurol. Res. 7: 24-26. 